\title{
Canonical Distribution Functions in Polymer Dynamics: I. Dilute Solutions of Flexible Polymers
}

\author{
Patrick Ilg,, , Iliya V. Karlin, ${ }^{2}$ and Hans Christian Öttinger ${ }^{2}$ \\ ${ }^{I}$ Institut für Theoretische Physik, Technische Universität Berlin, Hardenbergstr. 36, D-10623 Berlin, Germany \\ ${ }^{2}$ ETH Zürich, Department of Materials, Institute of Polymers, CH-8092 Zürich, Switzerland
}

(Dated: November 1, 2018)

\begin{abstract}
The quasi-equilibrium or maximum entropy approximation is applied in order to derive constitutive equations from kinetic models of polymer dynamics. It is shown in general and illustrated for an example how canonical distribution functions are obtained from the maximum entropy principle, how macroscopic and constitutive equations are derived therefrom and how these constitutive equations can be implemented numerically. In addition, a measure for the accuracy of the quasi-equilibrium approximation is proposed that can be evaluated while integrating the constitutive equations. In the example considered, it is confirmed that the accuracy of the approximation is increased by including more macroscopic variables. In steady elongational flow, it is found that more macroscopic variables need to be included above the coil-stretch transition to achieve the same accuracy as below.
\end{abstract}

PACS numbers: 83.80.Rs, 83.10.Gr, 05.20.Dd, 05.10.-a

\section{INTRODUCTION}

In this paper, the quasi-equilibrium or maximum entropy approximation is applied in order to derive constitutive equations from kinetic models of polymer dynamics. It is shown in general and illustrated for an example how canonical distribution functions are obtained from the maximum entropy principle, how macroscopic and constitutive equations are derived therefrom and how these constitutive equations can be implemented numerically. In addition, a measure for the accuracy of the quasiequilibrium approximation is proposed that can be evaluated while integrating the constitutive equations.

Polymer dynamics is a fascinating subject with important applications to polymer processing and turbulent drag reduction. Very recently, direct observation of the dynamics of individual DNA molecules in shear and elongational flow have become possible [1, 2, 3]. The experimental results provide useful information to improve our understanding of polymer dynamics in a flow. Since deformed polymer molecules cause stresses that are responsible for the macroscopic non-Newtonian rheological behavior of polymer solutions, polymer dynamics in the presence of a flow field is also of great interest from an application point of view.

Simple mechanical models are very helpful in this respect. Comparison of simulation results with experiments have shown that kinetic models of polymer molecules describe very well the rheological behavior of polymer fluids [4, 5] as well as the polymer conformation [6] in homogeneous shear and elongational flow. However, the use of these kinetic models is too timeconsuming to be used in numerical flow calculations of polymer solutions. Therefore, simplified constitutive equations have been proposed in the literature that describe stresses that the polymers exert on the flow for a given deformation history (see for example [T] and references therein). So far, however, no satisfactory constitutive equations are available that give accurate predictions under various flow conditions.

Kinetic models of polymer dynamics may serve as starting point for the derivation of constitutive equations. In general, constitutive equations cannot be derived exactly from kinetic theory due to the so-called closure problem. In addition, closure approximations have serious effects on the behavior of the model, and sometimes even lead to unphysical predictions [8]. Recently, the idea of using a set of canonical distribution functions (CDF) for obtaining closures to the kinetic equation have been proposed in [9] 10]. Motivated by the ease of computation, very special choices for the CDF are considered in [9, 10].

In the present work, CDF are introduced using the maximum entropy principle. This approach, also known as quasiequilibrium approximation, is also used successfully in other branches of statistical physics [11, 12]. The resulting, quasiequilibrium approximation to the dynamics, by construction, shows several desirable features like conservation of the dissipative nature of the dynamics and conservation of positive-definiteness of distribution functions. We show how this approximation can be used to obtain closed constitutive equations for polymer solutions.

We also present an algorithm for the numerical implementation of the constitutive equations. In addition, a measure for the accuracy of the approximation is suggested that does not require solutions to the kinetic model and can therefore be used while solving the constitutive equation. In the companion paper [13], this approach is applied to the kinetic theory of liquid-crystalline polymers. See also [14] for more details.

\footnotetext{
*ilg@physik.tu-berlin.de
} 
This paper is organized as follows. Kinetic models of dilute polymer solutions are reviewed in Sec. II. The maximum entropy principle is employed in Sec. IIII in order to obtain canonical distribution functions (CDF) for polymer solutions. Some properties of the CDF are also discussed. Closed form constitutive equations are derived using CDF with special emphasize on so-called conformation tensor models. A numerical integration scheme for the constitutive equations is presented in Sec. IV, In Sec. V, a measure for the accuracy of the approximate description of polymer dynamics with CDF is proposed. In Sec. VI, the derivation of constitutive equations using CDF together with the check of the accuracy is illustrated in detail for a kinetic model of polymer dynamics. Finally, some conclusions are offered in Sec. VII.

\section{KINETIC MODELS OF DILUTE POLYMER SOLUTIONS}

Let a linear, flexible polymer be represented by $N$ beads. The beads themselves represent many monomers, and are large compared to the solvent molecules. The distance between the beads is considered to be large enough so that it allows the random walk statistics for the neglected segments in between, but close enough to maintain these segments in equilibrium. The connectivity of the chain therefore is guaranteed by an entropic potential associated with the random walk statistics between the beads. Ideal random walk statistics corresponds to a harmonic potential, giving raise to linear forces acting on the beads. The forces acting on the beads are often pictured as springs, and the kinetic models are termed "bead-spring models".

\section{A. Kinetic Equations of Polymer Dynamics}

Equations of motion for bead-spring models can be found in most of the textbooks on polymer kinetic theory (see e.g. [15, 16]). Let $\mathbf{r}_{i}, i=1, \ldots, N$, denote the position vector of the $i$-th bead. The internal conformation of the chain is described by the connector vectors $\mathbf{Q}_{i}=\mathbf{r}_{i+1}-\mathbf{r}_{i}, i=1, \ldots, N-1$. In the classical approach, the polymer chain is exposed to a given homogeneous flow field $\mathbf{v}(\mathbf{r} ; t)=\mathbf{v}_{0}(t)+\boldsymbol{\kappa}(t) \cdot \mathbf{r}$, where $\boldsymbol{\kappa}(t)=\left(\nabla_{\mathbf{r}} \mathbf{v}(\mathbf{r} ; t)\right)^{\mathrm{T}}$ denotes the velocity gradient. Usually, incompressible flows, $\operatorname{Tr} \kappa=0$, are considered. The configurational distribution function $\Psi$ at time $t$ is assumed to depend on the internal conformation but to be independent of the location of the center of mass, $\Psi=\Psi\left(\left\{\mathbf{Q}_{i}\right\} ; t\right)$, where $\left\{\mathbf{Q}_{i}\right\}$ denotes the set of all connector vectors $\left\{\mathbf{Q}_{i}\right\}=\left\{\mathbf{Q}_{1}, \ldots, \mathbf{Q}_{N-1}\right\}$. The famous diffusion equation for $\Psi$ reads

$$
\frac{\partial \Psi}{\partial t}=J(\Psi)
$$

with

$$
J(\Psi)=-\sum_{i=1}^{N-1} \nabla_{\mathbf{Q}_{i}} \cdot\left\{\boldsymbol{\kappa} \cdot \mathbf{Q}_{i} \Psi\right\}+\frac{1}{2} \sum_{i, j=1}^{N-1} \nabla_{\mathbf{Q}_{i}} \cdot \hat{\mathbf{D}}_{i j} \cdot\left\{\left[\nabla_{\mathbf{Q}_{j}} U\right] \Psi+\nabla_{\mathbf{Q}_{j}} \Psi\right\},
$$

where $\nabla_{\mathbf{Q}}$ is the derivative with respect to $\mathbf{Q}$. The last term describes diffusion due to the collisions of the solvent molecules with the beads. Collisions keep each bead in thermal equilibrium with the solvent. Temperature, Boltzmann's constant and friction coefficient of the beads are denoted by $T, k_{\mathrm{B}}$ and $\zeta$, respectively. The function $U\left(\left\{\mathbf{Q}_{i}\right\}\right)$ is the dimensionless potential of forces on the beads so that $\mathbf{F}_{i}\left(\left\{\mathbf{Q}_{i}\right\}\right)=k_{\mathrm{B}} T \nabla_{\mathbf{Q}_{i}} U$ is the force acting on the $i$-the bead. It is generally assumed that only adjacent beads interact, the interaction being isotropic, so that $U$ can be written as

$$
U\left(\left\{\mathbf{Q}_{i}\right\}\right)=\sum_{i=1}^{N-1} V\left(\mathbf{Q}_{i}^{2}\right) .
$$

Long range interactions along the chain, such as those leading to excluded-volume effects might require some extra treatment. The diffusion matrix $\hat{\mathbf{D}}_{i j}$ can be represented as $\hat{\mathbf{D}}_{i j}=D\left(A_{i j}^{\mathrm{R}} \mathbf{1}+\zeta \hat{\Omega}_{i j}\right)$, where the diffusion constant $D$ is given by $D=2 k_{\mathrm{B}} T / \zeta$. The unit matrix is denoted by 1 , and the $(N-1) \times(N-1)$ Rouse matrix is

$$
A_{i j}^{\mathrm{R}}=2 \delta_{i j}-\left(\delta_{i, j+1}+\delta_{i, j-1}\right) .
$$

The tensors $\hat{\Omega}_{i j}\left(\left\{\mathbf{Q}_{i}\right\}\right)$ describe hydrodynamic interactions of bead $i$ and $j$ as the movement of the beads disturbs the flow field. Typically, the hydrodynamic interactions are described by (regularized) Oseen-Burger tensors [16]. The first term on the right hand side of Eq. (2) accounts for the hydrodynamic drag the beads experience while moving through the flow. It is assumed that the drag force is proportional to the difference of bead and solvent velocity.

The number density of polymers $n_{\mathrm{p}}=\langle 1\rangle$ is conserved by the dynamics (2). Here and in the sequel, brackets $\langle\bullet\rangle$ denote averages with respect to $\Psi,\langle\bullet\rangle=\int d^{d} Q \bullet \Psi\left(\left\{\mathbf{Q}_{i}\right\}\right)$ with $d=3(N-1)$. Eqs. (11) and (2) describe an ensemble of noninteracting bead-spring chains in the presence of a flow field. Therefore, these models describe the dynamics of dilute polymer 
solutions. The Rouse model, for example, is obtained when hydrodynamic interactions are neglected, $\hat{\Omega}_{i j}=\mathbf{0}$, and harmonic potentials $V\left(\mathbf{Q}^{2}\right)=H \mathbf{Q}^{2} /\left(2 k_{\mathrm{B}} T\right)$ are considered, $H$ denotes the spring constant. However, harmonic potentials are unrealistic, since they allow infinite stretching of the chain in strong flows. Warner's finite extensible nonlinear elastic potential (FENE),

$$
V\left(Q^{2}\right)=-(b / 2) \ln \left(1-Q^{2} / Q_{\max }^{2}\right),
$$

exhibits a logarithmic divergence at the maximum length $Q_{\max }$, and therefore prohibits further stretching [17]. The finite extensibility parameter, $b=H Q_{\max }^{2} /\left(k_{\mathrm{B}} T\right)$, is a dimensionless measure of the maximum extension. The Hookean spring force is recovered in the limit $b \rightarrow \infty$.

We restrict ourselves here to finite extensibility effects with or without hydrodynamic interactions. Other effects such as excluded volume, internal viscosity, friction coefficient varying with extension or anisotropic frictional drag can also be included in bead-spring models (see [15] and references therein).

\section{B. Entropy Production}

In the absence of flow, the distribution function $\Psi$ relaxes to its unique equilibrium state, $\Psi^{\mathrm{eq}}=e^{-U} / Z$, due to the dynamics (1) and (2), where $Z$ is the normalization constant. The Boltzmann entropy functional,

$$
S[\Psi]=-k_{\mathrm{B}} \int d^{d} Q \Psi\left(\left\{\mathbf{Q}_{i}\right\}\right) \ln \left[\Psi\left(\left\{\mathbf{Q}_{i}\right\}\right) / \Psi^{\mathrm{eq}}\left(\left\{\mathbf{Q}_{i}\right\}\right)\right]
$$

is strictly non-decreasing during the relaxation due to the dynamics (11) and (2) for $\boldsymbol{\kappa}=\mathbf{0}$. The entropy production $\dot{S}$ is defined as the time evolution of the entropy functional (6),

$$
\dot{S}=\int d^{d} Q \frac{\delta S[\Psi]}{\delta \Psi\left(\left\{\mathbf{Q}_{i}\right\}\right)} J(\Psi)
$$

where $\delta / \delta \Psi$ is the Volterra functional derivative. According to Eqs. (2) and (7), the entropy production can be split in two parts, $\dot{S}=\dot{S}^{h}+\dot{S}^{\text {d }}$, corresponding to the first and second term on the right hand side of Eq. (2), respectively. It is easily verified that $S$ is non-decreasing in the absence of flow,

$$
\dot{S}^{\mathrm{d}}=\frac{k_{\mathrm{B}}}{2} \sum_{i, j=1}^{N-1} \int d^{d} Q\left(\nabla_{\mathbf{Q}_{i}} \frac{\delta S[\Psi]}{\delta \Psi}\right) \cdot \hat{\mathbf{D}}_{i j} \Psi \cdot\left(\nabla_{\mathbf{Q}_{j}} \frac{\delta S[\Psi]}{\delta \Psi}\right) \geq 0
$$

if the diffusion matrix $\hat{\mathbf{D}}$ is positive semi definite. In the presence of a flow field, the entropy exchange $\dot{S}^{h}$ of the polymer and the solvent subsystem is given by

$$
\dot{S}^{h}=-\frac{1}{T} \kappa: \tau^{\mathrm{p}}
$$

which is not sign-definite. In Eq. (9), the polymer contribution to the stress tensor $\tau^{\mathrm{p}}$ has been introduced which is given by Kramer's expression [15, 16,

$$
\boldsymbol{\tau}^{\mathrm{p}}[\Psi]=(N-1) n_{\mathrm{p}} k_{\mathrm{B}} T \mathbf{1}-k_{\mathrm{B}} T \sum_{i=1}^{N-1} \int d^{d} Q \Psi\left(\left\{\mathbf{Q}_{i}\right\}\right) \mathbf{Q}_{i} \nabla_{\mathbf{Q}_{i}} U\left(\left\{\mathbf{Q}_{i}\right\}\right) .
$$

\section{CANONICAL DISTRIBUTION FUNCTIONS IN POLYMER DYNAMICS}

Within kinetic theory, the state of the polymer at time $t$ is described by the configurational distribution function $\Psi\left(\left\{\mathbf{Q}_{i}\right\} ; t\right)$ and Eqs. (1), (2) and (10) specify the dynamic and viscoelastic behavior of dilute polymer solutions for a given homogeneous flow field. In viscoelastic flow calculations, the velocity field has to be found numerically together with the dynamics of the polymers. The combined simulation of Navier-Stokes equation with the kinetic equation (1) is however very time-consuming. Therefore, there is an enormous interest in deriving closed constitutive equations from the kinetic equation. In general, this derivation cannot be done rigorously due to the so-called closure problem and certain approximations have to be invoked. 


\section{A. The Closure Problem}

It is assumed that the macroscopic behavior of the polymers can be described by a set of $n$ macroscopic variables, which are defined as functionals of the distribution function, $M_{k}=\hat{\Pi}_{k}[\Psi], k=1, \ldots, n$. We consider macroscopic variables that are linear functionals (moments) of the distribution function $\Psi$

$$
M_{k}(t)=\int d^{d} Q m_{k}\left(\left\{\mathbf{Q}_{i}\right\}\right) \Psi\left(\left\{\mathbf{Q}_{i}\right\} ; t\right) .
$$

The set of macroscopic variables need not be identical with the components of the stress tensor $\tau^{\mathrm{p}}$. We only require that the stress tensor $(10)$ is determined by the values of the macroscopic variables $\underline{M}=\left\{M_{1}, \ldots, M_{n}\right\}$. From the chain rule of differentiation and Eq. (1), the time evolution of $M_{k}$ is given by

$$
\dot{M}_{k}=\int d^{d} Q m_{k}\left(\left\{\mathbf{Q}_{i}\right\}\right) J(\Psi)
$$

which do not constitute, in general, a closed set of equations for the macroscopic variables. In what will follow, we present a general approach to obtain closures of Eqs. (12) based on the maximum entropy principle and corresponding canonical distribution functions.

\section{B. Maximum Entropy Principle and Canonical Distribution Functions}

The reduced or macroscopic description of the system is reasonable if a separation of time scales of the relaxation times holds. The equilibrium distribution function $\Psi^{\mathrm{eq}}=e^{-U} / Z$ is obtained from the maximum of the entropy functional (6) subject to fixed normalization, $1=\int d^{d} Q \Psi\left(\left\{\mathbf{Q}_{i}\right\}\right)$. The so-called generalized canonical distribution functions $\Psi^{*}$ are obtained by the maximum entropy principle under fixed constraints [12, 18, 19, 20],

$$
S[\Psi] \rightarrow \max , \quad M_{k}=\int d^{d} Q m_{k}\left(\left\{\mathbf{Q}_{i}\right\}\right) \Psi\left(\left\{\mathbf{Q}_{i}\right\}\right) .
$$

Solution to the problem Eq. (13), if it exists, is unique for concave entropy functionals like (6) and linear constraints given by Eq. (11). For the present case the solution to Eq. (13) is given explicitly as

$$
\Psi^{*}\left(\left\{\mathbf{Q}_{i}\right\}\right)=\Psi^{\mathrm{eq}}\left(\left\{\mathbf{Q}_{i}\right\}\right) \exp \left[\sum_{l=1}^{n} \Lambda_{l} m_{l}\left(\left\{\mathbf{Q}_{i}\right\}\right)+\Lambda_{0}\right]
$$

and is called canonical distribution function (CDF) (or generalized canonical state or quasi-equilibrium distribution function or maximum entropy state). The Lagrange multipliers, $\underline{\Lambda}=\left\{\Lambda_{0}, \Lambda_{1}, \ldots, \Lambda_{n}\right\}$, are determined from the constraints

$$
n_{\mathrm{p}}=\int d^{d} Q \Psi^{\mathrm{eq}}\left(\left\{\mathbf{Q}_{i}\right\}\right) \exp \left[\sum_{l=1}^{n} \Lambda_{l} m_{l}\left(\left\{\mathbf{Q}_{i}\right\}\right)+\Lambda_{0}\right]
$$

and

$$
M_{k}=\int d^{d} Q m_{k}\left(\left\{\mathbf{Q}_{i}\right\}\right) \Psi^{\mathrm{eq}}\left(\left\{\mathbf{Q}_{i}\right\}\right) \exp \left[\sum_{l=1}^{n} \Lambda_{l} m_{l}\left(\left\{\mathbf{Q}_{i}\right\}\right)+\Lambda_{0}\right]
$$

In most cases, the dependence $\underline{\Lambda}(\underline{M})$ or $\underline{M}(\underline{\Lambda})$ is not known explicitly. The manifold $\Omega^{*}$ that is spanned by $\Psi^{*}$ when the macroscopic variables take on all admissible values is known as quasi-equilibrium manifold or canonical distribution function manifold [21, 22].

The macroscopic entropy $S^{*}(\underline{M})$ is defined as the entropy $S[\Psi]$ given by Eq. (6) evaluated on the CDF $(14), S^{*}(\underline{M})=S\left[\Psi^{*}\right]$. The macroscopic entropy $S^{*}$ allows to interpret the Lagrange multipliers as the conjugate or dual to the macroscopic variables,

$$
\Lambda_{k}=-\frac{\partial S^{*}(\underline{M})}{\partial M_{k}} .
$$




\section{Macroscopic dynamics}

In what will follow, the canonical distribution function manifold $\Omega^{*}$ is used to obtain the macroscopic description of the polymer dynamics. We assume that arbitrary initial states relax quickly towards the manifold $\Omega^{*}$ and further evolve in the neighborhood of $\Omega^{*}$. In this case, the CDFs that span the manifold can be considered as representative states in the sense that moments of the solution to the kinetic equation (11) are approximated accurately by the corresponding moments evaluated with the CDFs. The validity of this assumption is closely related to the separation of time scales mentioned above and the choice of the macroscopic variables. This assumption can be interpreted within the context of the method of invariant manifold [21] as $\Omega^{*}$ being a meaningful approximation to the relevant invariant manifold. A measure of the accuracy of this approximation is presented in Sec. V. For improvements on this approximation see [21, 22]. We do not provide any rigorous proofs for the results obtained in the sequel. However, the ability of measuring the accuracy of the approximation is a constructive method to gain confidence in the approximation scheme proposed here.

Having identified the manifold $\Omega^{*}$, the macroscopic time evolution is defined as

$$
\dot{M}_{k}^{*}=\int d^{d} Q m_{k}\left(\left\{\mathbf{Q}_{i}\right\}\right) J\left(\Psi^{*}\left(\underline{M},\left\{\mathbf{Q}_{i}\right\}\right)\right),
$$

which is the dynamics of the macroscopic variables (12) evaluated on $\Omega^{*}$. Inserting the right hand side of Eq. (2) for $J$, Eq. (18) becomes

$$
\dot{M}_{k}^{*}=\boldsymbol{\kappa}^{\mathrm{T}}: \sum_{i=1}^{N-1}\left\langle\mathbf{Q}_{i} \nabla_{\mathbf{Q}_{i}} m_{k}\right\rangle_{\Lambda}-\sum_{l=1}^{n} \hat{M}_{k l} \Lambda_{l},
$$

where $\langle\bullet\rangle_{\Lambda}$ denotes averages with respect to the CDFs $\Psi^{*}$, Eq. (14), and $\hat{M}$ is a positive semi-definite matrix,

$$
\hat{M}_{k l}=\frac{1}{2} \sum_{i, j=1}^{N-1} \int d^{d} Q\left(\nabla_{\mathbf{Q}_{i}} m_{k}\right) \cdot \hat{\mathbf{D}}_{i j} \Psi^{*} \cdot\left(\nabla_{\mathbf{Q}_{j}} m_{l}\right) .
$$

Eq. (19) together with Eqs. (15), (16) and (20) represent the set of closed equations describing the macroscopic dynamics. Since the CDFs are assumed to be representative distributions, the constitutive relation is obtained by evaluating Eq. 10 ) on $\Omega^{*}$, $\tau^{\mathrm{p} *}(\underline{M})=\tau^{\mathrm{p}}\left[\Psi^{*}\right]$. In the present case it reads

$$
\tau^{\mathrm{p}^{*}}(\underline{M})=-k_{\mathrm{B}} T \sum_{i=1}^{N-1} \sum_{k=1}^{n}\left\langle\mathbf{Q}_{i} \nabla_{\mathbf{Q}_{i}} m_{k}\right\rangle_{\Lambda} \Lambda_{k} .
$$

From Eqs. (17) and (19) it is found that the macroscopic entropy production $\dot{S}^{*}$ is given by

$$
\dot{S}^{*}=-\frac{1}{T} \kappa: \tau^{\mathrm{p} *}+\sum_{k, l=1}^{n} \Lambda_{k} \hat{M}_{k l} \Lambda_{l} .
$$

and could have been obtained by evaluating Eqs. (8) and (9) on the CDF (14). In the absence of flow, the macroscopic entropy production (22) is positive semi-definite as is the underlying kinetic model, Eq. (8). This illustrates a general property of the quasi-equilibrium approximation that it conserves the type of dynamics (see, e.g. [22]).

\section{Conformation Tensor Models}

In the present section, we specialize to conformation tensors as macroscopic variables. The corresponding conformation tensor models are frequently used in the literature [23]. The conformation tensors $\sigma$ are the covariance matrices of the distribution function

$$
\boldsymbol{\sigma}_{i j}=\left\langle\mathbf{Q}_{i} \mathbf{Q}_{j}\right\rangle, \quad i, j=1, \ldots, N-1 .
$$

In our notation, conformation tensor models are defined by the special choice $M_{k} \rightarrow \boldsymbol{\sigma}_{i j}$ and $m_{k} \rightarrow \mathbf{Q}_{i} \mathbf{Q}_{j}$ in Eq. (11). Corresponding Lagrange multipliers are denoted by $\boldsymbol{\Lambda}_{i j}$.

For conformation tensor models the time evolution equation (19) reads

$$
\dot{\boldsymbol{\sigma}}_{i j}=\boldsymbol{\kappa} \cdot \boldsymbol{\sigma}_{i j}+\boldsymbol{\sigma}_{i j} \cdot \boldsymbol{\kappa}^{\mathrm{T}}-\sum_{k, l=1}^{n} \hat{\mathbf{M}}_{i j k l}: \boldsymbol{\Lambda}_{k l},
$$


where the matrix (20) now reads

$$
\hat{\mathbf{M}}_{i j k l}=\frac{1}{2}\left\langle\mathbf{Q}_{i} \hat{\mathbf{D}}_{j k} \mathbf{Q}_{l}+\mathbf{Q}_{i} \hat{\mathbf{D}}_{j l} \mathbf{Q}_{k}+\mathbf{Q}_{j} \hat{\mathbf{D}}_{i k} \mathbf{Q}_{l}+\mathbf{Q}_{j} \hat{\mathbf{D}}_{i l} \mathbf{Q}_{k}\right\rangle_{\Lambda} .
$$

In Eq. (24) one recognizes the upper-convected derivative, while the last term describes relaxation mechanisms.

For the present choice of macroscopic variables, the stress tensor (21) takes the form

$$
\boldsymbol{\tau}^{\mathrm{p}}=-2 k_{\mathrm{B}} T \sum_{i, j=1}^{N-1} \boldsymbol{\sigma}_{i j} \cdot \boldsymbol{\Lambda}_{j i}
$$

\section{NUMERICAL INTEGRATION SCHEME}

In the maximum entropy principle (13), Lagrange multipliers $\underline{\Lambda}$ occur naturally to satisfy the constraints. In principle, the Lagrange multipliers can be expressed as functions of the macroscopic variables using the constraints (15) and (16). In general, however, no explicit expressions $\underline{\Lambda}(\underline{M})$ can be found. For these cases, we here propose a method that does not require knowledge of the functions $\underline{\Lambda}(\underline{M})$

\section{A. Dual Variables and Their Dynamics}

A change of the independent variables from the set $\underline{M}$ to $\underline{\Lambda}$ is done by Legendre transform. The Legendre transform of the macroscopic entropy $S^{*}(\underline{M})$ is the generating function $G(\underline{\Lambda})=S^{*}(\underline{M})+\sum_{k} \Lambda_{k} M_{k}$, which can be written as

$$
G(\underline{\Lambda})=\ln \int d^{d} Q \Psi^{\mathrm{eq}}\left(\left\{\mathbf{Q}_{i}\right\}\right) \exp \left[\sum_{l=1}^{n} \Lambda_{l} m_{l}\left(\left\{\mathbf{Q}_{i}\right\}\right)\right] .
$$

The macroscopic variables and higher order moments can be obtained by derivatives of $G$ with respect to $\Lambda$,

$$
M_{k}(\underline{\Lambda}) \equiv\left\langle m_{k}\right\rangle_{\Lambda}=\frac{\partial G(\underline{\Lambda})}{\partial \Lambda_{k}}
$$

and

$$
C_{k l}(\underline{\Lambda}) \equiv\left\langle m_{k} m_{l}\right\rangle_{\Lambda}-\left\langle m_{k}\right\rangle_{\Lambda}\left\langle m_{l}\right\rangle_{\Lambda}=\frac{\partial^{2} G(\underline{\Lambda})}{\partial \Lambda_{k} \partial \Lambda_{l}}
$$

The Lagrange multipliers $\underline{\Lambda}$ are now considered as primary independent variables that parameterize the CDFs (14) and that determine the values of the macroscopic variables (28). From Eqs. (28) and (29) it is found that the time evolution of $\underline{\Lambda}$ is given by

$$
\dot{\Lambda}_{k}=\sum_{l=1}^{n} C_{k l}^{-1}(\underline{\Lambda}) \dot{M}_{l}(\underline{\Lambda})
$$

where $\dot{M}_{k}(\underline{\Lambda})$ is given by Eq. (18). Eq. (30) is the closed macroscopic equation written in dual variables. Note, that the constitutive relation (21) is already given as a function of $\underline{\Lambda}$.

\section{B. Numerical Integration Scheme}

The reformulation of the macroscopic dynamics (19) in terms of the dual variables $\underline{\Lambda}$ described in Sec. IV A is suitable for numerical implementation. The Lagrange multipliers $\underline{\Lambda}$ now play the role of independent dynamic variables, instead of $\underline{M}$. To advance given values $\underline{\Lambda}(t)$ at time $t$ to their values $\underline{\Lambda}(t+\tau)$, with small time step $\tau$, the following first order integration scheme is proposed [24]:

1. The new values of the macroscopic variables $\underline{M}(t+\tau)$ are found from the values $\underline{\Lambda}(t)$ by

$$
\frac{M_{k}(t+\tau)-M_{k}(t)}{\tau}=\int d^{d} Q m_{k}\left(\left\{\mathbf{Q}_{i}\right\}\right) J\left(\Psi^{*}\left(\underline{\Lambda}(t),\left\{\mathbf{Q}_{i}\right\}\right)\right)
$$

where $J\left(\Psi^{*}\left(\underline{\Lambda}(t),\left\{\mathbf{Q}_{i}\right\}\right)\right)$ denotes the right hand side of Eq. (2) $)$, evaluated with the $\operatorname{CDF}(14)$ with $\underline{\Lambda}(t)$ the actual values of the Lagrange multipliers. 
2. The matrix $C_{k l}$, defined in Eq. (29), is evaluated from

$$
C_{k l}(t)=\left\langle m_{k} m_{l}\right\rangle_{\Lambda}(t)-M_{k}(t) M_{l}(t),
$$

where $\left\langle m_{k} m_{l}\right\rangle_{\Lambda}$ is calculated from Eq. (31), where $m_{k}$ is now replaced by $m_{k} m_{l}$.

3. The $n \times n$ matrix $\mathbf{C}(t)$ is inverted numerically to give $C^{-1}(t)$.

4. The values of the Lagrange multipliers, $\underline{\Lambda}(t+\tau)$, are given by

$$
\frac{\Lambda_{k}(t+\tau)-\Lambda_{k}(t)}{\tau}=\sum_{l=1}^{n} C_{k l}^{-1}(t) \frac{M_{k}(t+\tau)-M_{k}(t)}{\tau}
$$

This concludes one time step of integration. The integration scheme has to be supplemented by initial conditions $\underline{\Lambda}(0)$. The special case of equilibrium initial conditions corresponds to $\underline{\Lambda}(0)=\underline{0}$.

Note, that evaluating the matrix (29) requires moments of $\Psi$ which are of higher order than the macroscopic variables themselves. If $n$ denotes the number of macroscopic variables, the numerical integration scheme requires in total $n(n+3) / 2$ integrals per time step to evaluate $\underline{M}$ and $\mathbf{C}$. Due to the symmetry of $\mathbf{C}$, this number is of order $n^{2} / 2$ rather than $n^{2}$. The evaluation of $Y_{k}$ may require additional integrals. The evaluation of all these integrals with standard numerical methods might be timeconsuming, especially for high-dimensional integrals. It is demonstrated in [25] that under certain circumstances these integrals can be evaluated efficiently by adapting methods of fast Fourier transformations.

\section{ACCURACY OF APPROXIMATION BY CANONICAL DISTRIBUTION FUNCTIONS}

The reduced description outlined in Sec. III obviously imposes restrictions or approximations to the underlying kinetic model. Therefore, the accuracy of the reduced description needs to be discussed. The convergence of arbitrary initial conditions to the manifold $\Omega^{*}$ is of obvious importance, but is in general difficult to study. For a concrete example, the convergence or non-convergence is investigated in [26].

CDFs are in general not solutions to the kinetic equation (1), i.e. the canonical distribution function subspace $\Omega^{*}$ is not dynamically invariant. The dynamic variance $\Delta$ is defined as

$$
\Delta(\underline{M})=J\left(\Psi^{*}\right)-\sum_{k=1}^{n} \frac{\partial \Psi^{*}}{\partial M_{k}} \dot{M}_{k}
$$

which is the difference between the microscopic time evolution evaluated on $\Omega^{*}$ and the time evolution of the CDFs due to the macroscopic dynamics. The dynamic variance $\Delta$ can be considered as 'defect' of the macroscopic description. While the method of invariant manifold [21] uses $\Delta$ to obtain improved manifolds, we will later use $|\Delta|$ as a measure of the accuracy of the approximation by CDFs. The norm $|\bullet|$ used later is related to $\Delta_{\ell}$, which is the difference of the dynamics of moment $M_{\ell}$ due to the microscopic and macroscopic dynamics,

$$
\Delta_{\ell} \equiv \int d^{d} Q m_{\ell} \Delta=\frac{d}{d t}\left\langle m_{\ell}\right\rangle_{\Lambda}-\left[\left\langle m_{\ell} m_{j}\right\rangle_{\Lambda}-\left\langle m_{\ell}\right\rangle_{\Lambda}\left\langle m_{j}\right\rangle_{\Lambda}\right]\left(C^{-1}\right)_{j k} \dot{M}_{k}
$$

$\Delta_{\ell}$ vanishes identically for $\ell \leq n$ as it has to. For $\ell>n$, the quantities $\left\langle m_{\ell}\right\rangle_{\Lambda}$ are not included in the list of macroscopic variables (13). If these quantities are defined such that the set $\left\{\underline{M},\left\langle m_{\ell}\right\rangle_{\Lambda}\right\}$ is linear independent of $\underline{M}$, Eq. (35) provides valuable information on the dynamics of variables that are not included in the list of macroscopic variables.

Inserting in Eq. (34) the right hand side of Eq. (2) for $J$ and Eq. (14) for $\Psi^{*}$ together with the time evolution of the macroscopic variables (19) one obtains $\Delta_{\underline{M}}=\Psi^{*} \hat{\Delta}_{\underline{M}}$ with

$$
\begin{aligned}
& \hat{\Delta}_{\underline{M}}=-\operatorname{Tr} \boldsymbol{\kappa}-\sum_{i=1}^{N-1} \mathbf{Q}_{i} \cdot \boldsymbol{\kappa}^{\mathrm{T}} \cdot\left(-\nabla_{\mathbf{Q}_{i}} U+\boldsymbol{\xi}_{i}^{0}\right)-\sum_{i, k=1}^{N-1} \boldsymbol{\kappa}:\left\langle\left[\nabla_{\mathbf{Q}_{i}} m_{k}\right] \mathbf{Q}_{i}\right\rangle_{\Lambda} \xi_{k}^{1} \\
& \quad+\frac{1}{2} \sum_{i, j=1}^{N-1} \nabla_{\mathbf{Q}_{i}} \cdot \hat{\mathbf{D}}_{i j} \cdot \boldsymbol{\xi}_{j}^{0}-\frac{1}{2} \sum_{i, j, k=1}^{N-1}\left(\left\langle\nabla_{\mathbf{Q}_{j}} \hat{\mathbf{D}}_{i j}\left[\nabla_{\mathbf{Q}_{i}} m_{k}\right]\right\rangle_{\Lambda}-\left\langle\left[\nabla_{\mathbf{Q}_{i}} m_{k}\right] \hat{\mathbf{D}}_{i j} \nabla_{\mathbf{Q}_{j}} U\right\rangle_{\Lambda}\right) \xi_{k}^{1} .
\end{aligned}
$$

In Eq. (36), we have defined $\boldsymbol{\xi}_{i}^{0}=\sum_{k} \Lambda_{k} \nabla_{\mathbf{Q}_{i}} m_{k}$ and $\xi_{k}^{1}=\partial \ln \Psi^{*} / \partial M_{k}$. For the special case of conformation tensors as the macroscopic variables the expression (36) simplifies on the replacement $\underline{M} \rightarrow \mathbf{Q Q}$. If in addition one specializes to certain mean-field potentials, then the expression for the variance becomes most transparent. This is demonstrated in the sequel. 


\section{A. Gaussian Manifolds of Conformation Tensor Models}

In this section we consider general potentials of the form

$$
U\left(\left\{\boldsymbol{\sigma}_{i j}\right\}\right)=\sum_{i=1}^{N-1} V\left(\operatorname{Tr} \boldsymbol{\sigma}_{i i}\right)
$$

for the kinetic equation (1). The restriction to these potentials is very important. They can either be viewed as mean-field approximations to an underlying potential of the form (3) or as models in their own right. Concerning the first interpretation, a possible derivation together with systematic corrections to this approximation is illustrated in [22].

For conformation tensor models, the equilibrium states are isotropic Gaussian distributions if the potentials are of the form (37). An important property of conformation tensor models for these potentials is that also the quasi-equilibrium states (14) are Gaussian. In this particular case, Eq. (23) can be evaluated by Gaussian integration and subsequently be inverted to give the Lagrange multipliers as explicit functions of the macroscopic variables

$$
\boldsymbol{\Lambda}_{i j}\left(\left\{\boldsymbol{\sigma}_{i j}\right\}\right)=V^{\prime}\left(\operatorname{Tr} \boldsymbol{\sigma}_{i i}\right) \mathbf{1} \delta_{i j}-\frac{1}{2}\left(\boldsymbol{\sigma}^{-1}\right)_{i j}
$$

Inserting the functional form of the Lagrange multipliers $(38)$ and the potential $(37)$ into the quasi-equilibrium distribution function we obtain the standard representation of the symmetric Gaussian distribution functions

$$
\Psi \boldsymbol{\sigma}\left(\left\{\mathbf{Q}_{i}\right\}\right)=\frac{1}{\sqrt{(2 \pi)^{d} \operatorname{det} \boldsymbol{\sigma}}} \exp \left\{-\frac{1}{2} \sum_{i, j=1}^{N-1} \mathbf{Q}_{i} \cdot\left(\boldsymbol{\sigma}^{-1}\right)_{i j} \cdot \mathbf{Q}_{j}\right\}
$$

Up to an irrelevant constant, also the quasi-equilibrium entropy $S^{*}(\boldsymbol{\sigma})$ is found from Eq. (38) as explicit function of $\boldsymbol{\sigma}$,

$$
S^{*}\left(\left\{\boldsymbol{\sigma}_{i j}\right\}\right)=\frac{1}{2} \ln \operatorname{det} \boldsymbol{\sigma}-U\left(\left\{\boldsymbol{\sigma}_{i j}\right\}\right) .
$$

The first term on the right hand side of Eq. (40) originates from the normalization of $\Psi_{\boldsymbol{\sigma}}$ while the second term contains the entropic spring potential.

\section{B. Variance of Conformation Tensor Models}

For potentials of the form (37), the time evolution equation for the conformation tensor $\sigma$ is obtained from Eqs. (24), 25) and (38) and reads

$$
\dot{\boldsymbol{\sigma}}_{i j}=\boldsymbol{\kappa} \cdot \boldsymbol{\sigma}_{i j}+\boldsymbol{\sigma}_{i j} \cdot \boldsymbol{\kappa}^{\mathrm{T}}-\sum_{k=1}^{N-1} V^{\prime}\left(\operatorname{Tr} \boldsymbol{\sigma}_{k k}\right)\left\langle\mathbf{Q}_{i} \hat{\mathbf{D}}_{j k} \cdot \mathbf{Q}_{k}+\hat{\mathbf{D}}_{i k} \cdot \mathbf{Q}_{k} \mathbf{Q}_{j}\right\rangle_{\Lambda}+\left\langle\hat{\mathbf{D}}_{i j}\right\rangle_{\Lambda}
$$

where use has been made of the decomposition of moments for the Gaussian distribution functions (see e.g. Appendix A of [27]). It is not surprising, that Eq. (41) coincides with the result of the Gaussian approximation used in [27, the averages being performed with the Gaussian distribution (39). Eq. (41) is an obvious generalization of Eq. (5) in [27] for the anharmonic potentials of the form (37). In Eq. (41) the "incompressibility" condition $\nabla \cdot \hat{\mathbf{D}}=\mathbf{0}$ for hydrodynamic interaction tensors is assumed.

The variance of the QEA (36) can be calculated for the kinetic equation (1) by Gaussian integrals. The result is

$$
\Delta_{\boldsymbol{\sigma}}=\Psi \boldsymbol{\sigma}\left[-\sum_{i, j=1}^{N-1} \operatorname{Tr}\left(\mathbf{T}_{i j} \cdot\left(\boldsymbol{\sigma}^{-1}\right)_{j i}\right)+\sum_{i, j, k, l=1}^{N-1}\left(\left(\boldsymbol{\sigma}^{-1}\right)_{i k} \cdot \mathbf{Q}_{k}\right)^{\mathrm{T}} \cdot \mathbf{T}_{i j} \cdot\left(\left(\boldsymbol{\sigma}^{-1}\right)_{j l} \cdot \mathbf{Q}_{l}\right)\right]
$$

where we have introduced the tensor $\mathbf{T}_{i j}=\mathbf{T}_{i j}^{0}+\mathbf{T}_{i j}^{1}$, with the contribution of the Brownian part as $\mathbf{T}_{i j}^{0}=\hat{\mathbf{D}}_{i j}-\left\langle\hat{\mathbf{D}}_{i j}\right\rangle_{\Lambda}$ and the spring contribution as $\mathbf{T}_{i j}^{1}$,

$$
\mathbf{T}_{i j}^{1}=\sum_{k=1}^{N-1} V^{\prime}\left(\operatorname{Tr} \boldsymbol{\sigma}_{k k}\right)\left[\left\langle\hat{\mathbf{D}}_{i l} \cdot \mathbf{Q}_{l} \mathbf{Q}_{j}\right\rangle_{\Lambda}-\hat{\mathbf{D}}_{i l} \cdot \boldsymbol{\sigma}_{l j}\right]
$$


We will see in a moment why the flow term does not contribute to $\Delta \sigma$.

In the absence of hydrodynamic interactions or for pre- or self-consistently averaged hydrodynamic interactions, the diffusion matrix $\hat{\mathbf{D}}$ is independent of $\left\{\mathbf{Q}_{i}\right\}$. Upon inspection of the variance $\sqrt[42]{ }$, one notices it immediately that in these cases the variance $\Delta_{\boldsymbol{\sigma}}$ vanishes identically. In these case, the drift term in the diffusion equation (2) is linear in $\mathbf{Q}$ and the diffusion term is independent of $\mathbf{Q}$. In this case, Gaussian distributions provide exact solutions to the diffusion equation [28]. Examples are the Rouse and Zimm models but also models that incorporate finite extensibility effects in the Peterlin approximation. From the above discussion we learn that in this case the Gaussian distributions constitute the exact invariant manifold of the dynamics (11) and Eq. (41) represents the time evolution equation on the invariant manifold. Since there exist many solutions to Fokker-Planck equation the relevance of the Gaussian solutions is discussed in the next section.

For general hydrodynamic interactions the variance (42) does not vanish and the quasi-equilibrium approximation (39) is no longer exact. It may serve as initial approximation as, for example in [27]. Possible improvements on the QEA can be found in [22].

\section{ILLUSTRATION}

As we have already mentioned, the choice of macroscopic variables is not unique. Very different choices of macroscopic variables are possible, and correspond to different sets of microscopic states accessible to the macroscopic description. In the literature, conformation tensors are frequently used as macroscopic variables. In Sec. VB, it is shown for a particular class of models, that the quasi-equilibrium manifold for these variables is the invariant manifold. In case the invariant manifold is stable, one observes a fast relaxation to the invariant manifold followed by a slow evolution on the manifold.

In general, the quasi-equilibrium manifold is not invariant and improvements on this approximation become an important issue. If the set of macroscopic variables is maintained, successive improvements on the QEA can be found along the lines of the method of invariant manifolds [21]. Another option is to extend the set of macroscopic variables and apply the QEA for the larger set of variables. Here, we illustrate the latter option for the kinetic model of polymer dynamics considered in [29].

In the context of polymer dynamics, the problem of the choice of macroscopic variables is encountered frequently. Recent experiments of $\mathrm{Chu}$ and co-workers [1] clearly demonstrate the dependence of the polymer dynamics in flow on the initial state. A possible classification of initial states into dumbbell, half-dumbbell, folded, coiled and kinked conformations is proposed in [5]. The failure of the macroscopic description in terms of a single conformation tensor has attracted considerable interest and led de Gennes to the notion of 'molecular individualism' [30].

In order to deal with this situation, the macroscopic description requires an extended set of variables. In [9, 10], the addition of one extra variable associated to the fourth moment of the distribution function is proposed. In [9, 10], also a closure approximation for this set of variables is proposed. In this closure, however, a penalty function has to be introduced ad hoc in order to obtain physically admissible results.

Here, we study the macroscopic description for an extended set of variables in the QEA. As demonstrated in [12], the QEA is well-behaved for any choice of macroscopic variables. In the limit where the number of macroscopic variables goes to infinity, the microscopic description is recovered since no variation over microscopic states is performed in the maximum entropy principle and therefore no reduction of description is associated with the QEA. We illustrate here for a finitely extensible dumbbell model that the macroscopic description converges fast with increasing number of macroscopic variables. We consider the one-dimensional model proposed in [29], with the spring force

$$
F(Q)=H Q_{1} \tan \left(Q / Q_{1}\right), \quad \text { for }-Q_{\max }<Q<Q_{\max }
$$

where $Q_{1}=2 Q_{\max } / \pi$ is determined by the maximum extension $Q_{\max }$ and $H$ denotes the "spring constant" in the sense $F(Q) \rightarrow H Q$ for $Q \rightarrow 0$. The force (44) approximates the inverse Langevin function very well and allows the exact calculation of the spectrum of relaxation times of the associate Fokker-Planck operator. The result is

$$
\lambda_{n}=2 \lambda_{\mathrm{H}}\left[n+n^{2} / b_{1}\right]^{-1}, \quad n=1,2, \ldots
$$

where $\lambda_{\mathrm{H}}=\zeta /(4 H)$ is the time constant of the Hookean dumbbell and $b_{1}=H Q_{1}^{2} / k_{\mathrm{B}} T$ a dimensionless finitely extensibility parameter 29]. Also the exact eigenfunction have been found in [29] to be given by $\Psi_{n}=\Phi_{n} \Psi_{\text {eq }}^{1 / 2}$ with

$$
\Phi_{n}(Q)=C_{n} a_{0}^{\dagger} a_{1}^{\dagger} \ldots a_{n-1}^{\dagger} \cos ^{n+b_{1} / 2}\left(Q / Q_{1}\right)
$$

where $a_{k}^{\dagger}=-\nabla_{Q}+f_{\alpha_{k}}$ are generalized creation operators. $C_{n}$ denote normalization constants and $f_{\alpha_{k}}=\alpha \tan \left(Q / Q_{1}\right)$. The first eigenfunctions read: $\Psi_{0}=\Psi_{\text {eq }}, \Psi_{1}=N_{1} \sin \left(Q / Q_{1}\right) \Psi_{\text {eq }}, \Psi_{2}=N_{2}\left[\left(b_{1}+1\right) \sin ^{2}\left(Q / Q_{1}\right)-\cos ^{2}\left(Q / Q_{1}\right)\right] \Psi_{\text {eq }}$ with $N_{1}^{2}=b_{1}+2$ and $N_{2}^{2}=\left(b_{1}+4\right) /\left[2\left(b_{1}+1\right)\right]$. Note, that the eigenfunctions are orthonormal, $\left\langle\Psi_{n}, \Psi_{m}\right\rangle_{\mathrm{s}}=\delta_{n m}$, with respect 
to the scalar product

$$
\langle g, h\rangle_{\mathrm{s}}=\int_{-Q_{\max }}^{Q_{\max }} d Q \Psi_{\mathrm{eq}}^{-1}(Q) g(Q) h(Q)
$$

As macroscopic variables, choose the first $n$ even dimensionless moments of the distribution function $M_{k}=\left\langle\tilde{Q}^{2 k}\right\rangle_{\Lambda}, k=$ $1, \ldots, n$ and $\tilde{Q}=Q / Q_{1}$ is the reduced extension. The benefit of considering a one-dimensional model is of course that there is no tensorial structure associated with these moments. The QEA for this choice of macroscopic variables is of the form (14) and reads

$$
\Psi_{\Lambda}^{*}(Q)=\left(1 / z_{\mathrm{eq}}\right) \cos ^{b_{1}}(\tilde{Q}) \exp \left[\sum_{j=1}^{n} \Lambda_{j} \tilde{Q}^{2 j}+\Lambda_{0}\right]
$$

The dynamic equations for the macroscopic variables found from Eq. (19) are

$$
\dot{M}_{k}=2 k \kappa M_{k}+k(2 k-1) M_{k-1} /\left(b_{1} \lambda_{\mathrm{H}}\right)-k R_{k} / \lambda_{\mathrm{H}}
$$

where $R_{k}=\left\langle\tan (\tilde{Q}) \tilde{Q}^{2 k-1}\right\rangle_{\Lambda}$. The polymer contribution to the stress found from Kramer's expression $(10)$ is $\tau^{\mathrm{p}} /\left(n_{\mathrm{p}} k_{\mathrm{B}} T\right)=$ $1-b_{1} R_{1}$.

For given values of the $n$ Lagrange multipliers at time $t, \underline{\Lambda}(t)$, the corresponding values of the $n$ macroscopic variables are found from

$$
M_{k}(t)=\frac{2 e^{\Lambda_{0}(t)}}{z_{\mathrm{eq}}} \int_{0}^{\pi / 2} d \tilde{Q} \tilde{Q}^{2 k} \cos ^{b_{1}}(\tilde{Q}) \exp \left[\sum_{j=1}^{n} \Lambda(t) \tilde{Q}^{2 j}\right]
$$

Thanks to the finite extensibility of the model, the integration in Eq. (50) is performed over a closed interval only. In addition, due to the symmetry of the integrand, the integration is performed over the positive range of $\tilde{Q}$ only. The matrix $C_{k l}=\left\langle\tilde{Q}^{2(k+l)}\right\rangle_{\Lambda}$ is obtained by the $n(n+1) / 2$ corresponding integrals. In this particular case, some of these integrals are identical to components of $\underline{M}$. Inversion of the matrix $\mathbf{C}$ is facilitated by the fact that it is a symmetric, positive semi-definite matrix. In total, one time step of integration requires $n(n+5) / 2$ one-dimensional integrals similar to Eq. (50). The numerical integrations were performed using standard integration schemes such as Gauss-Legendre or Romberg integration [31]. The algorithm, described above and in Sec. IV B was implemented and run for fixed time step $\tau$ in the order of $10^{-2} \ldots 10^{-4} \lambda_{\mathrm{H}}$.

In particular, we consider equilibrium initial conditions $\left(\Lambda_{k}(t=0)=0\right)$ and steady flows and compare the quasi-equilibrium dynamics for different numbers $n$ of macroscopic variables. We generally expect the QEA to be a good approximation near the true equilibrium. As noted in Sec. IIID, the approximation (48) is exact in the steady state since the one-dimensional analogue of the conformation tensor is kept as macroscopic variables and any flow in one dimension is a potential flow. Therefore, we expect (48) to be a good approximation also near the steady state.

In Fig. 1], the polymer stress $\tau^{\mathrm{p}} /\left(n_{\mathrm{p}} k_{\mathrm{B}} T\right)$ is shown as a function of dimensionless time $t / \lambda_{\mathrm{H}}$ for equilibrium initial conditions and different strengths of the flow. The finite extensibility parameter was chosen to be $b=50$. Details of the numerical integration of Eq. (49) and evaluation of $R_{k}$ and $\tau^{\mathrm{p}}$ with the distribution function (48) are given in Sec. IV B. As seen from Fig. 1 a), we observe that for weak or moderately strong flows, $\hat{\kappa} \lesssim 0.5$, the analogue of the conformation tensor $(n=1)$ already provides a satisfactory description. Within the resolution of Fig. 1 a), the predictions are the same also for higher values of $n$. We observe that the description in terms of the second moment becomes worse in stronger flows, $\hat{\kappa}>0.5$ Including higher order moments, we observe a fast convergence of the polymer stress in the macroscopic description to the result of the microscopic simulation. As seen in Fig. 1 b), the first three even moments already provide a satisfactory description for $\hat{\kappa}=1$.

It is interesting to trace back the difficulties of the macroscopic description in terms of second moments to the behavior of the probability distribution function (pdf) in these flows. Since the macroscopic description corresponds to the manifold of distribution functions (48), direct comparison between microscopic and macroscopic description is possible also on the level of distribution functions. In Fig. 2, the quasi-equilibrium states (48) for the corresponding values of the Lagrange multipliers are compared to the numerical solutions of the kinetic equation at time $t=2 \lambda_{\mathrm{H}}$ and $t=8 \lambda_{\mathrm{H}}$. In the present case, the kinetic equation is not solved directly but the stochastic differential equation associated with the kinetic equation is solved numerically for a large ensemble of dumbbells [16]. This method is well-established and is known as Brownian dynamics simulation. In this case, the distribution function is represented by a histogram over the lengths of the dumbbells of the ensemble. As seen from Fig. 2 a), the QEA for $n=1$ gives a good approximation even for the distribution function in case the elongational flow is not too strong, $\hat{\kappa} \lesssim 0.5$. In stronger flows, the shape of the distribution function is not accurately predicted by the quasi-equilibrium distribution (48) for $n=1$, both, for rather early times (circles) and later times (diamonds). A much better approximation of the distribution function for earlier times is obtained for $n=2$. Note, that for later times a characteristic peak of the distribution function near the maximum extension occurs [ 8$]$, indicating that most dumbbells are stretched to almost their maximum length. 
Note, that the precise shape of that peak is not described perfectly by the quasi-equilibrium distributions even for $n=3$. The approximation is however reasonably close to give accurate predictions for the polymer stress as seen in Fig. 11.

As discussed in Sec. V, the accuracy of the approximation of the dynamics by canonical distribution functions can be measured by a suitable norm of the dynamic variance $\Delta$. Let us reconsider the approximation (48) with $n=1$. Then, the quantity $\Delta_{2}$ defined in Eq. (35) ) for $\ell=2$ measures the difference of the microscopic and macroscopic dynamics of the fourth moment. For two strengths of the flow, $\Delta_{2}$ is given as a function of time in Fig. 3. From Fig. 3 one notices that $\Delta_{2}$ vanishes for early times $t / \lambda_{\mathrm{H}} \ll 1$ since the initial state was chosen to be the equilibrium distribution for which $\Delta_{2}=0$. Also for times $t \gg 1$, the dynamic variance $\Delta_{2}$ vanishes, since the steady state is correctly described by the approximation (48). For intermediate times $t \approx 1, \Delta_{2}$ is different from zero and can be interpreted as a measure for the quality of the approximation. For $\hat{\kappa}=0.5$, $\left|\Delta_{2}\right|$ remains small, less than $10^{-3}$, while for $\hat{\kappa}=1.0,\left|\Delta_{2}\right|$ is two orders of magnitude larger. This is in agreement with the comparison to Brownian dynamics simulations shown in Fig. 1 that the approximation $n=1$ performs quite well for $\hat{\kappa}=0.5$, whereas it is much less reliable for $\hat{\kappa}=1.0$. Note that $\left|\Delta_{2}\right|$ is a measure for the difference of the microscopic and macroscopic time evolution. Therefore, the maximum of $\left|\Delta_{2}\right|$ occurs at earlier times than the maximum difference of the moments and the polymer stress.

Although the model studied in this section is only one-dimensional, we expect the basic observations to be valid also in three dimensions. The present approach can easily be generalized to three dimensions and it would be interesting to confirm this conjecture. Remember that the quasi-equilibrium distributions (48) are exact in equilibrium and in the steady state in case of potential flows. Therefore, it would be particularly interesting to see whether the above results also apply in case of non-potential flows in three dimensions.

It is straightforward to apply the numerical integration scheme proposed in Sec. IV B to the three-dimensional problem. The corresponding numerical integrations are however much more time consuming than in the one dimension. Under certain circumstances, these integrals may be evaluated efficiently, e.g. by fast Fourier transform algorithms [25].

Based on the observations for this model, we conclude this section with the remark that the frequently used conformation tensor models might not perform very well in strong flows if compared to the original model. In the present example, the failure of the conformation tensor model is related to the so-called 'coil-stretch transition' [32], giving raise also to the instability of the invariant manifold as studied in [26].

\section{CONCLUSION}

In the present work, we have shown how the quasi-equilibrium or maximum entropy approximation can be applied to kinetic models of polymer dynamics. As a result, canonical distribution functions and constitutive equations of polymer dynamics are obtained. A numerical implementation of the constitutive equations is proposed together with a measure for the accuracy of the quasi-equilibrium approximation. The numerical implementation and the measure of the accuracy of the approximation is illustrated in detail for an example of polymer dynamics. It is found that the accuracy of the approximation increases with increasing number of macroscopic variables. In steady elongational flow, more macroscopic variables are needed in order to arrive at the same accuracy above the coil-stretch transition than below.

Finally, we mention that the same methods can be applied to other kinetic models of polymer dynamics like, e.g. liquidcrystalline polymers [13].

[1] T. T. Perkins, D. E. Smith, S. Chu, Single Polymer Dynamics in an Elongational Flow, Science 276 (1997) $2016-2021$.

[2] D. E. Smith, S. Chu, Response of Flexible Polymers to a Sudden Elongational Flow, Science 281 (1998) 1335-1340.

[3] D. E. Smith, H. P. Babcock, S. Chu, Single-Polymer Dynamics in Steady Shear Flow, Science 283 (1999) 1724-1727.

[4] L. Li, R. G. Larson, Comparison of Brownian Dynamics Simulations with Microscopic and Light Scattering Measurements of Polymer Deformation under Flow, Macromolecules 33 (2000) 1411-1415.

[5] L. Li, R. G. Larson, T. Sridhar, Brownian Dynamics Simulations of Dilute Polystyrene Solutions, J. Rheol. 44 (2000) $291-322$.

[6] J. S. Hur, E. S. G. Shaqfeh, R. G. Larson, Brownian Dynamics Simulations of Single DNA Molecules in Shear Flow, J. Rheol. 44 (2000) 713-742.

[7] R. B. Bird, J. M. Wiest, Constitutive Equations for Polymeric Liquids, Ann. Rev. Fluid Mech. 27 (1995) 169-193.

[8] R. Keunings, On the Peterlin Approximation for Finitely Extensible Dumbbells, J. Non-Newton. Fluid Mech. 68 (1997) 85-100.

[9] G. Lielens, P. Halin, I. Jaumin, R. Keunings, V. Legat, New Closure Approximations for the Kinetic Theory of Finitely Extensible Dumbbells, J. Non-Newton. Fluid Mech. 76 (1998) 249-279.

[10] G. Lielens, R. Keunings, V. Legat, The FENE-L and FENE-LS Closure Approximation to the Kinetic Theory of Finitely Extensible Dumbbells, J. Non-Newton. Fluid Mech. 87 (1999) 279-296.

[11] R. Balian, From Microphysics to Macrophysics. Vol. 2: Methods and Applications of Statistical Physics, Springer, Berlin, 2 nd Ed., 1992.

[12] A. N. Gorban, Equilibrium Encircling, Chapt. 3, Quasi-Equilibrium Entropy Maximum, pp. 92-122, Nauka, Novosibirsk, 1984. 
[13] P. Ilg, I. V. Karlin, H. C. Öttinger, Canonical Distribution Functions in Polymer Dynamics: Liquid-Crystalline Polymers, (2001), in preparation.

[14] P. Ilg, Reduced Description of Kinetic Models of Polymer Dynamics, Wissenschaft \& Technik Verlag, Berlin, 2001.

[15] R. B. Bird, C. F. Curtiss, R. C. Armstrong, O. Hassager, Dynamics of Polymeric Liquids, Vol. 2, Kinetic Theory, Wiley, New York, 1987.

[16] H. C. Öttinger, Stochastic Processes in Polymeric Fluids, Springer, Berlin, 1996.

[17] H. R. Warner, Kinetic Theory and Rheology of Dilute Suspensions of Finitely Extensible Dumbbells, Ind. Eng. Chem. Fundamentals 11 (1972) 379-387.

[18] G. W. Gibbs, Elementary Principles of Statistical Mechanics, Dover, New York, 1960.

[19] E. T. Jaynes, Papers on Probability, Statistics and Statistical Physics, ed. R. D. Rosenkranz, Creidel, Dordrecht, 1983.

[20] D. Zubarev, V. Morozov, G. Röpke, Statistical Mechanics of Nonequilibrium Processes, Akademie Verlag, Berlin, 1997.

[21] A. N. Gorban, I. V. Karlin, Method of Invariant Manifolds and Regularization of Acoustic spectra, Transp. Theor. Stat. Phys. 23 (1994) 559-632.

[22] A. N. Gorban, I. V. Karlin, P. Ilg, H. C. Öttinger, Corrections and Enhancements of Quasi-Equilibrium States, J. Non-Newton. Fluid Mech. 96 (2001) 203-219.

[23] M. Dressler, B. J. Edwards, H. C. Öttinger, Macroscopic Thermodynamics of Flowing Polymeric Liquids, Rheol. Acta 38 (1999) 117-136.

[24] P. Ilg, I. V. Karlin, H. C. Öttinger, Reconstruction of Constitutive Equations from Brownian Dynamics Proc. XIII Int. Congress on Rheology (Cambridge, 2000) Vol. 2, 64-66.

[25] J. E. Hurtado, A. B. Barbat, Fourier-based Maximum Entropy Method in Stochastic Dynamics, Structural Safety 20 (1998) $221-235$.

[26] P. Ilg, I. V. Karlin, Validity of a Macroscopic Description in Dilute Polymeric Solutions, Phys. Rev. E 62 (2000) 1441-1443.

[27] H. C. Öttinger, Gaussian Approximation for Rouse Chains with Hydrodynamic Interaction, J. Chem. Phys. 90 (1988) $463-473$.

[28] H. C. Öttinger, A Model of Dilute Polymer Solutions with Hydrodynamic Interaction and Finite Extensibility. I. Basic Equations and Series Expansions, J. Non-Newton. Fluid Mech. 26 (1987) 207-446.

[29] P. Ilg, I. V. Karlin, S. Succi, Supersymmetry Solution to Finitely Extensible Dumbbell Model, Europhys. Lett. 51 (2000) $355-360$.

[30] P. G. de Gennes, Molecular Individualism, Science 276 (1997) 1999-2000.

[31] W. H. Press, S. A. Teukolsky, W. T. Vetterling, B. P. Flannery, Numerical Recipes in FORTRAN: The Art of Scientific Computing, Cambridge University Press, Cambridge, 1992.

[32] P. G. de Gennes, Coil-Stretch Transition of Dilute Flexible Polymers under Ultrahigh Velocity Gradients, J. Chem. Phys. 60 (1974) 5030-5042. 

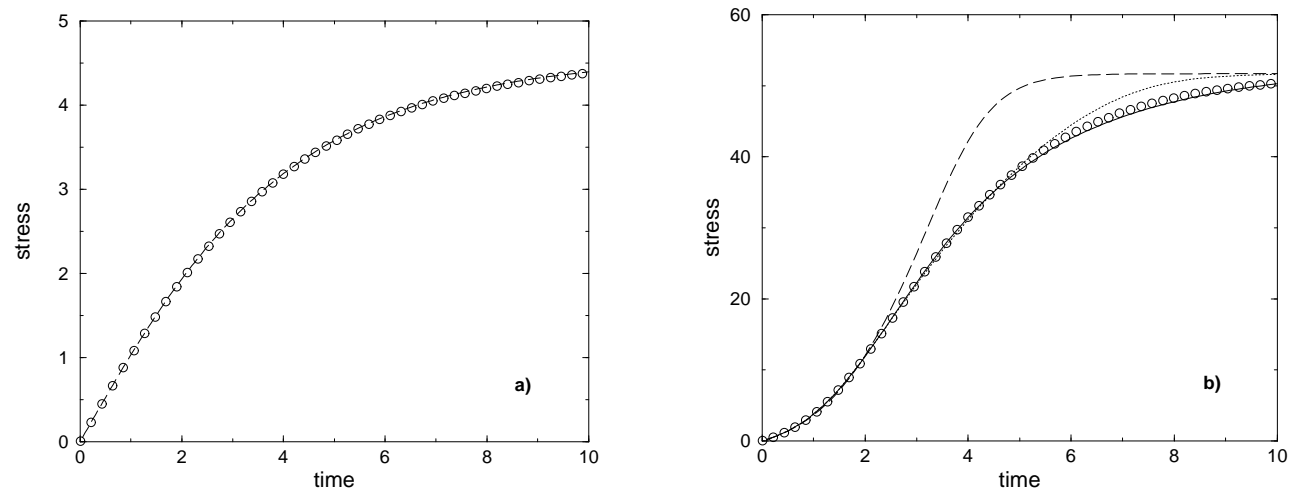

FIG. 1: Performance of QEA in steady elongational flow with $\hat{\kappa}=0.5$ a) and $\hat{\kappa}=1.0 \mathrm{~b}$ ). Shown is the polymer stress $\tau^{\mathrm{P}}$ in units of $n_{\mathrm{p}} k_{\mathrm{B}} T$ as a function of dimensionless time $t / \lambda_{\mathrm{H}}$ for different sets of macroscopic variables. Dashed: $n=1$, dotted: $n=2$, solid: $n=3$ and symbols: Brownian dynamics simulations of full kinetic model. 

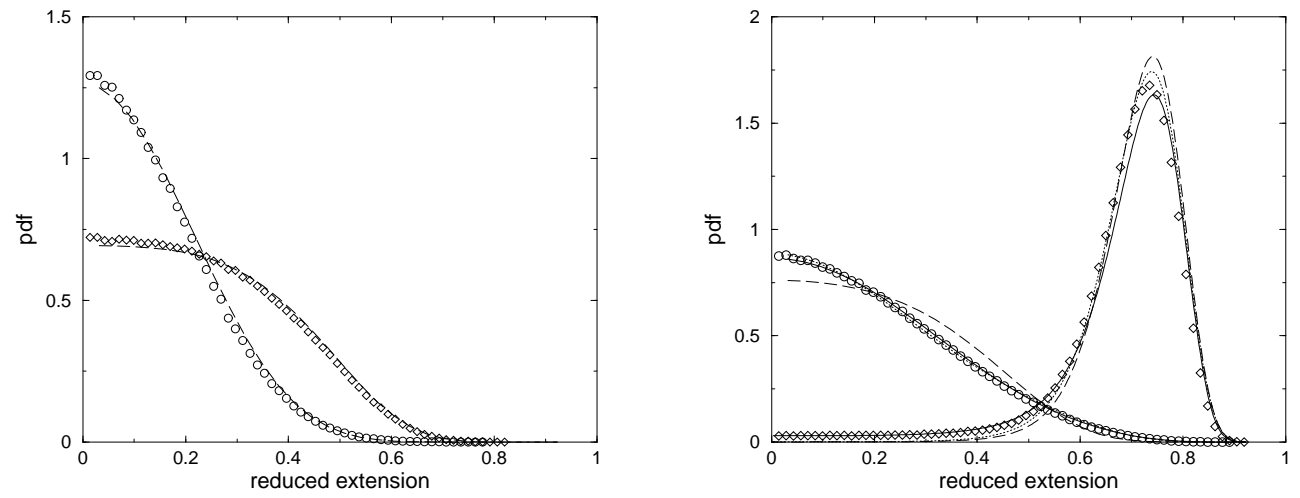

FIG. 2: Performance of QEA in steady elongational flow with $\hat{\kappa}=0.5$ a) and $\hat{\kappa}=1.0 \mathrm{~b}$ ). Shown is the distribution function as a function of reduced extension $Q / Q_{\max }$ for different sets of macroscopic variables at two times. Dashed: $n=1$, dotted: $n=2$, solid: $n=3$ and symbols: Brownian dynamics simulations of full kinetic model. 


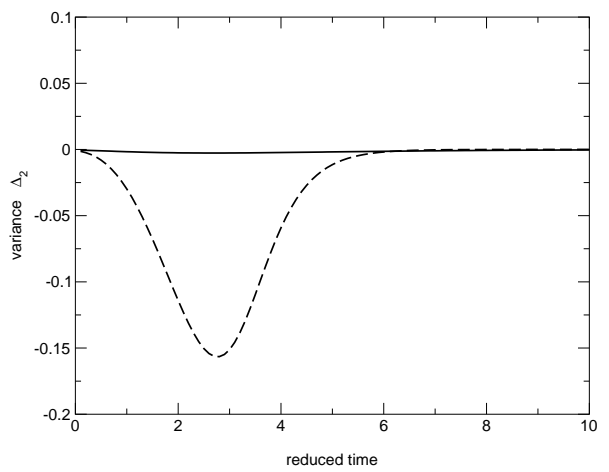

FIG. 3: Dynamic variance $\Delta_{2}$ as a function of reduced time $t / \lambda_{\mathrm{H}}$. Solid line corresponds to $\hat{\kappa}=0.5$, broken line to $\hat{\kappa}=1.0$. Comparison to Fig. 1 shows that the dynamic variance $\Delta_{2}$ can be used as a measure for the accuracy of the approximation. 